\title{
Preceptorship scheme for newly qualified physician associates working in general practice in Sheffield
}

\author{
Authors: Julie Hoskin ${ }^{A}$ and Ria Agarwal ${ }^{B}$
}

\begin{abstract}
We describe a preceptorship model that was initiated in March 2018 for newly qualified physician associates (PAs) in primary care in Sheffield, UK. The scheme enabled part funding for four band 6 preceptorship posts initially, alongside fortnightly teaching and mentorship by the preceptorship lead and a senior PA external to the employer. The number of posts had increased to 20 at the time of writing, due to the success of the scheme. We discuss how the scheme arose, and feedback from the general practice (GP) supervisors and the preceptorship PAs. The advantages of an external senior GP PA mentoring the preceptorship PA is highlighted, and this scheme may be the first of its kind to initiate this level of mentorship. A 'preceptorship scale' is suggested, which may aid employers in thinking about in-house support for this new member of their team in their first year of practice.
\end{abstract}

KEYWORDS: Physician associate, preceptorship scale, GP PA, primary care, Faculty of Physician Associates

DOI: 10.7861/clinmed.2020-0221

\section{Introduction}

Previous literature has established that there are decreasing numbers of doctors and nurses in primary care. ${ }^{1}$ Based on a data collection exercise undertaken in 2017 (Fig 1), a significant number of general practitioners (GPs; 44 GP partners and salaried GPs out of a total of 401 , or almost $11 \%$ ) were considering retiring within the next 5 years. Regular bulletins from the local medical committees and the clinical commissioning group (CCG) suggested a vacancy rate of between 12 and 17 GP posts monthly from a total of 82 practices, and this provided an additional incentive to think of an alternative answer to the 'replacing like with like' approach most favoured by practices. Physician associates (PAs) represent an alternative answer, so efforts began to encourage interest in choosing a newly qualified PA to help with workforce issues. $^{2}$

Authors: ${ }^{\text {A }}$ general practice physician associate preceptorship lead, Sheffield Clinical Commissioning Group, Sheffield, UK and physician associate ambassador regional scheme lead, Health Education England, Sheffield, UK; ${ }^{\text {B }}$ physician associate, Sothall Medical Centre, Sheffield, UK and senior lecturer, Sheffield Hallam University, Sheffield, UK

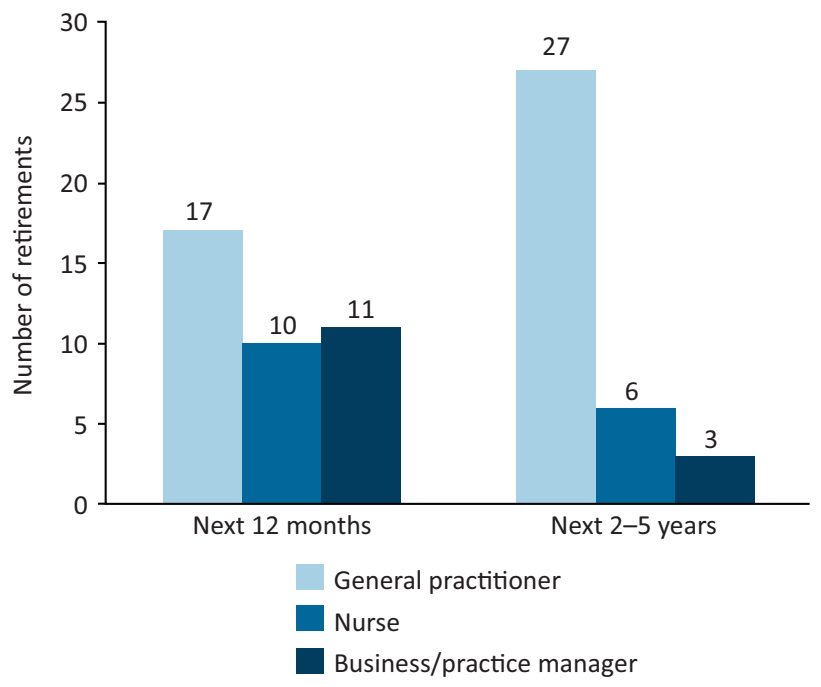

Fig 1. Retirements in the following 12 months and the following 2-5 years.

Yorkshire and Humber Health Education England (HEE; now part of North East and Yorkshire HEE) had also made a significant effort to promote the PA role in the region by encouraging higher education institutions (HEIs) to develop PA courses, paying the 2 years of postgraduate study fees and subsidising the PAs with a $£ 5,000-10,000$ bursary while studying. PA students from the two local HEIs had primary care exposure as part of their placement experience. Following this, interest was expressed by students and practices to consider creating GP PA roles (Fig 2). However, practices were concerned about investing in such a new member of the workforce when there were issues around the level of supervision required, and a known inability for PAs to formally prescribe due to lack of regulation. General practice teams are smaller, group dynamics are more fragile and financial risks are felt more personally. Thus, the challenge was getting the PAs into post so that the GPs could see them at work, become interested and take the initiative themselves, gradually building the workforce.

\section{Business proposal for preceptorship scheme}

The business proposal was predicated on the need to try to embed the PAs into the workforce so that they could demonstrate their worth once in post. Understanding the risk-averse culture 


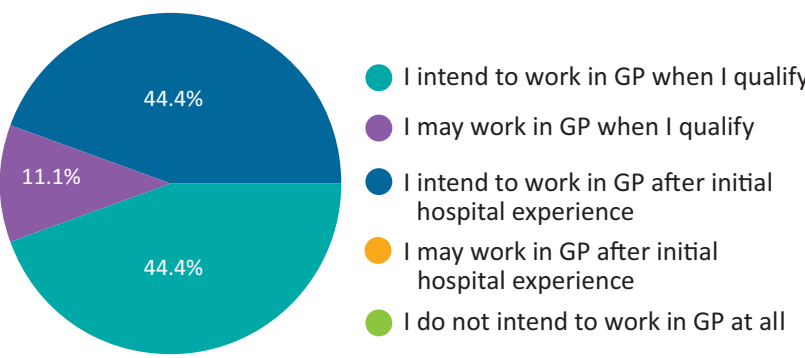

Fig 2. Results from a survey undertaken in November 2017 on Sheffield Hallam University's first physician associate cohort (due to qualify in January 2018) in response to the question 'When you qualify, how likely are you to work in primary care?' $n=18 . \mathrm{GP}=$ general practice.

of general practice and the preceptorship needs of the newly qualified PAs, we were convinced that offering a training grant to mitigate the time-consuming nature of the initial supervision and support for the PA would help to incentivise those practices which had long-standing GP vacancies to consider an alternative practitioner. The structure was decided upon by considering the preceptorship year in quarters, with the more concentrated supervision and support in the first 3 months, reducing commensurately during each following quarter. Locum costs if covering for the GP to support the PA, consultation times, the debrief model used for foundation year-2 doctors and GP registrars, and time to study were factored in. Recognising the need to pitch the training grant at a realistic level to attract attention, $£ 15,000$ was the highest value that we felt would be acceptable to GPs, the CCG and HEE, as the intention was to ask for match funding.

Consequently the CCG agreed to part-fund two PA band 6 posts initially, and HEE North East agreed to match this, so with four supported posts the next stage was introducing the PAs to the primary care community of Sheffield, who had not had a student placement and therefore had little knowledge of the PA role. A workforce workshop was organised in which presentations around allied health professions were delivered, and the local practices were invited to attend. The training grant scheme was advertised in the CCG bulletin. Eight practices applied for the four grants and all were awarded, based on a vacancy scoring system. Following a further request to $\mathrm{HEE}$ to fund the additional places, the remaining practices were also awarded a training grant and the Sheffield GP PA Preceptorship Scheme came into being. Starting small with five PAs appointed and commencing in March 2018, the eight places were soon filled, and data have consequently been gathered from these surgeries. Eligibility for the grant included a signed agreement to provide dedicated in-house support by a named GP supervisor, weekly half-day release for self-study or to attend the fortnightly structured support provided by the CCG, and permission to allow a senior PA (including one of the authors) to attend surgeries to provide a dedicated session of mentorship and observation.

\section{Methodology}

The fortnightly educational sessions, in which local doctors, PAs or nurses shared their expertise on a specific clinical area, were arranged depending on the educational requests of the PAs. A consultation observation session was offered to the preceptorship PA 6 months after commencement, and as a follow-up at 1 year. ${ }^{3}$ We used the Consultation Observation Tool (COT) developed for GP trainees.

Qualitative feedback was sought via Google surveys of the preceptorship PAs and their supervisors in spring 2020. The supervisors were asked about their views on the PA(s) in the team, the impact of the training grant, and their opinions of the value of the educational components. The PAs were also asked about their views on the preceptorship scheme, in addition to regular contact about what they would find helpful. The PAs were also asked to list their clinical duties (Fig 3) and supervisor support required, although these data were captured prior to the COVID-19 pandemic, in which the PAs have had to move towards telephone/ video consultations where possible. Participants were aware that these data have been collated with a view to dissemination.

\section{Results}

The PA results indicated that length of appointments varied as experience and confidence grew; many of the group are now providing 15-minute consultations, whereas the newer members are still on 30-minute consultations. Most indicated that they had a regular debrief, although those who had been in post for over a year tended to request this on an 'as and when' basis rather than on a fixed basis. Seventy-eight per cent valued the protected education sessions, and those who did not mainly identified that
Fig 3. Responses to the question 'Which of the following types of activities do you currently engage in?' $n=12$.

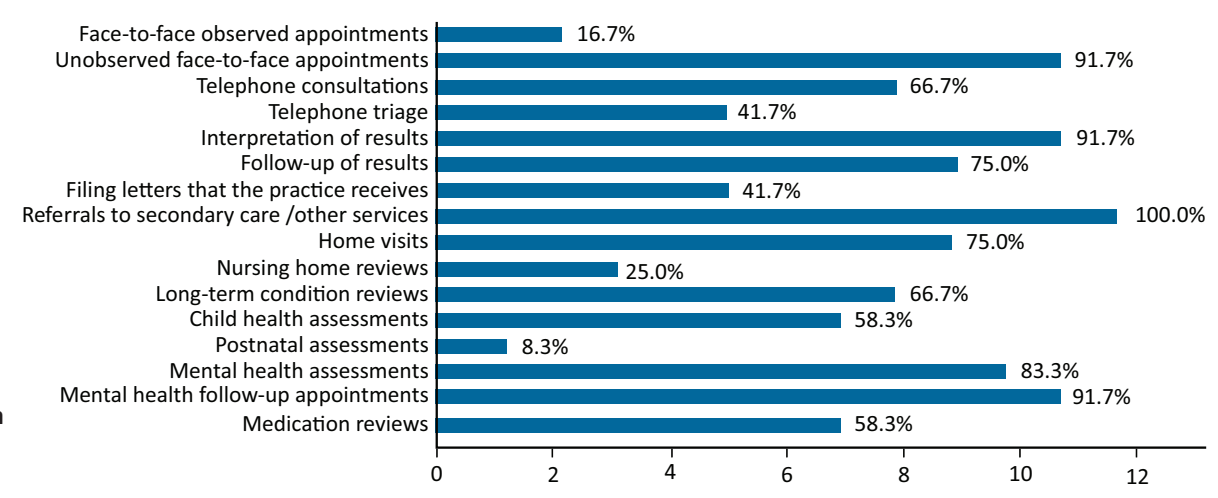


this was due to attending the same sessions at the start and end of their preceptorship year. Low numbers and lack of funding meant that offering two strands of education wasn't possible at this time. However, some PAs saw the session as a useful refresher, and still valued the protected time to network with each other, rather like a First5 group for newly qualified GPs. ${ }^{4}$ Consultation observation feedback from PAs was mainly positive, identifying that the process increased their confidence and they valued the feedback and support. Those who found it most helpful were PAs lacking a consistent in-house support system. Those who were observed without the COT said that they would have found this tool helpful for specific future development. Overall, they felt well supported to grow in scope and duties throughout their preceptorship period, which has likely contributed to the excellent retention rate in this scheme. The PAs stated that the debriefing and in-house discussions were invaluable for their learning and development.

Feedback from the GPs suggested that having the part-funding in place was a helpful incentive to consider the role for the first time, following initial reluctance around lack of prescribing rights and the supervision required. GP supervisor comments have been very positive, one highlighting that the PAs were "capable of far more than expected', were 'enthusiastic, willing, eager to learn and take on responsibility' and were able to see a wider range of presentations than advanced nurse practitioners (ANPs). Regarding the timetabled educational sessions, comments from GPs indicated that it appeared 'valuable and appropriate to primary care', and that having a forum for networking was a helpful concept that mimicked some GP vocational training schemes. While some of the preceptorship PAs did not share their consultation observation feedback with their supervisors, the GPs felt that it was a valuable idea, although some were not aware that this had occurred. The GPs have also reported positive feedback from patients, with some patients even requesting to see the PA instead of the GP.

\section{Discussion}

The feedback has been positive as a whole, and this scheme is especially innovative in providing mentorship from an experienced PA. In addition to identifying areas for improvement in terms of both consultation content and style through observing the infrastructure of the consultation, the senior PA can act as an external source of role-specific support to the newly qualified PA. This also doubles as a safety net for the conscientious GP supervisor, who may feel anxious about taking ownership of supervising a new member in their team. A systematic observation tool such as the COT may be a helpful additional document for salary negotiation and performance discussion at the end of the preceptorship year, and complements the paperwork provided by the Faculty of Physician Associates (FPA) through offering another perspective. ${ }^{5}$ The current paperwork from the FPA supports use of mini clinical evaluation exercise (mini-CEX) and observed procedural skills, both of which are more relevant to secondary care PAs.

As with any other clinician, appropriate support and supervision from their supervisor will allow the PA to be nurtured and grow clinically. However we found that, in a minority of cases, PAs did not progress as well through their preceptorship year as expected, leading to symptoms and signs similar to those of trainees in difficulty. ${ }^{6}$ This has led to our proposal of a GP PA preceptorship scale (Fig 4), which could be utilised by employers in the preceptorship year as a possible structure. The scale
20-30 minute appointments at start of preceptorship: $6-8$ slots per clinic

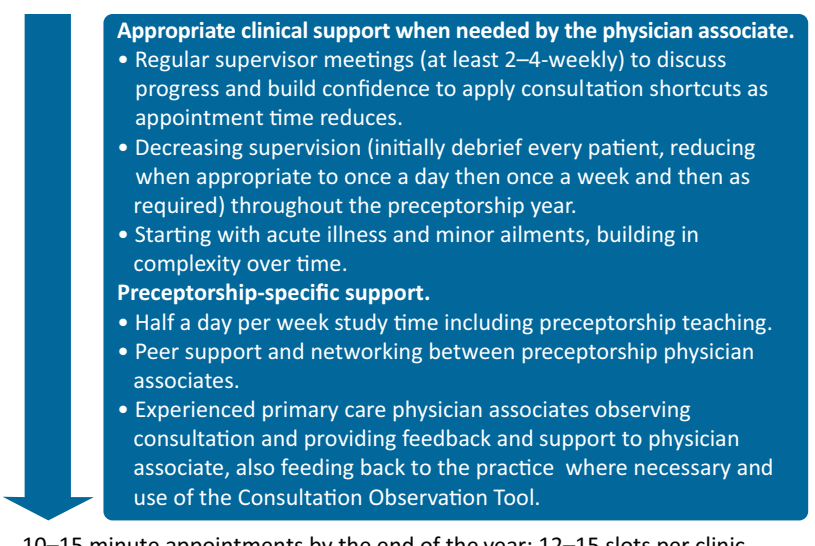

10-15 minute appointments by the end of the year: $12-15$ slots per clinic

\section{Fig 4. Suggested general practice physician associate preceptorship} scale.

creates a map of the PA's starting point versus where they would like them to be in a year, and mutual discussion is encouraged at agreed intervals through the year to explore the ways in which this can be achieved. Inhibition to progress down this scale in our experience was commonly due to three areas: lack of adequate in-house support, lack of access to appropriate tools and software, or the PA not feeling confident in applying 'consultation shortcuts' to streamline the consultation. Any of these issues were observed to trigger frequently running behind, which could lead to missing out on the preceptorship scheduled teaching, working less efficiently and possibly burnout. For example, debrief quality and frequency provided by the supervisor may not match the expectation of the newly qualified and probably rather anxious PA. Increased anxiety itself can be a factor that inhibits progression; the leap from student to practising healthcare worker is a rather large jump in any profession. This was also identified by one of the GPs, who highlighted that the PA she employed needed more support when they first started. Fortunately, this PA was patiently encouraged and supported by the supervising GP, enabling them to flourish. However, if not highlighted or addressed sensitively by the employer, this may also contribute to the individual not functioning as efficiently as they could, which in a PA's case may lead to job dissatisfaction and a move toward another specialty. ${ }^{7}$

Similarly, having no system in place to manage prescribing or advice from the duty doctor can lead to the PA feeling unsupported or contribute to delays in clinic time. These delays could then have negative connotations on how the PA views their role at the practice, alongside adding to stress for the PA and the waiting patient. An example of this was when a scheduled preceptorship session on streamlining consultations was conducted; we were interested to note that very few of the PAs who had been practising for several months were aware of how to organise electronic prescribing with their supervisor. Discussion then occurred at this stage to educate the PAs on how to manage this crucial area, which in practice will save the PA time. Issues such as these are also role specific; GP trainees and many ANPs are prescribers, therefore issues such as these may not be covered in house unless the surgery has experience of working with PAs. Having support in place will likely increase job satisfaction and 
thus retention, in addition to possibly attracting more PAs into the tricky terrain of primary care.

There has been a positive response from the GP supervisors and, where available, their perception of patient feedback, with most of the preceptorship PAs staying on at their destination surgeries following their preceptorship year. Several surgeries employed a second PA, with one even employing a third. This is consistent with previous literature around doctor satisfaction with the PA role. ${ }^{8}$ Numbers have grown steadily so that there are now 18 PAs in post, with one due to start in July 2020 and one student PA employed in an assisting role waiting to sit her national exam (postponed due to COVID-19). Most of these PAs are in post at 30-37.5 hours/week and there has been almost full PA retention; one has left primary care, but still attends preceptorship teaching and remains part of the community of practice. One PA has dropped some primary care hours to take up a part-time role in secondary care. The scheme also welcomes PA colleagues from the local mental health trust for shared learning, which unofficially raises the numbers to 27 .

The limitations of our results include the timing of data gathering; some PAs were newer to post than others who had been in post since 2018, so inexperienced PAs may have placed additional value on the support given. Some supervisors were also more familiar with the PA role than others, which may have led to heightened awareness of being able to 'benchmark' the progress of the preceptorship PA. Additionally the preceptorship PAs themselves varied in life experience, which may have impacted on the supervisor feedback. For example, one of the preceptorship PAs joined the scheme following several months' experience in secondary care. The small numbers within this scheme provide further limitations, although this is the case with most research into the role due to the relatively small numbers in this new profession. COVID-19 impacted the provision of consultation observation, so not all PAs were able to comment on this.

\section{Conclusion}

The CCG continues to promote the scheme by offering the training grant, providing one of the authors as the dedicated lead for the project, supporting both PAs and practices, and sharing the details of the scheme locally and regionally. The scheme has attracted PAs from out of the area, with some of the preceptorship PAs from Leeds and Birmingham, and other regions contacting us for vacancy information. The preceptorship PAs continue to develop their community of practice, supporting each other and the new joiners, and attending the preceptorship group teaching. Many of the PAs also aid support of PA students on placement from the local HEIs. Two of the group are Physician Associate Ambassadors Yorkshire \& Humber, promoting the role further. The group are also invited to support student PAs in the Sheffield Hallam University objective structured clinical examinations and are trained accordingly, thus also adding other skills to their portfolios.

\section{Next steps}

Previous literature has identified a 'need to explore the development of a common curriculum for development of PAs entering primary care for the first time'. ${ }^{9}$ We have proposed a 'preceptorship scale', and encourage use of it by all primary care PA supervisors to map the starting and completion points of their employed PA, rather than an unstructured approach. In PAs not progressing down the scale, observation of the consultations by a senior GP PA could be a helpful strategy in assessing how the PA manages their consultation, thus providing specific strategies for any issues arising. This may not be feasible for the practice themselves to coordinate due to time pressures, or they may feel that they lack role-specific insight. Use of a structured template such as the COT was noted to be particularly helpful in assessing consultation infrastructure in a systematic manner. The COT could be used widely for performance review alongside the appraisal and internship paperwork available from the FPA.

\section{Summary}

What is known?

While there is some literature on the benefits of PAs in primary care and the duties they can perform, this did not necessarily reassure local GPs, who were cautious about employing PAs in their own surgeries.

\section{What is the question?}

How could GPs be encouraged to make use of this new member of their workforce, and how would they integrate into the existing primary care team? Would a preceptorship model support nurturing and clinical development of the PA, in addition to in-house support from the practice?

\section{What was found?}

GPs valued the financial support from the preceptorship scheme, in addition to the educational support provided. The PAs mostly valued extra support from the scheme in addition to their existing in-house support. A 'preceptorship scale' for newly qualified PAs in primary care has been suggested for primary care employers to complement existing support and education.

\section{What is the implication for practice?}

If funding was available, this preceptorship scheme could be rolled out as a blueprint in regional 'pockets' where newly qualified GP PA numbers are high to enable more adequate rolespecific support.

\section{References}

1 Rimmer A. Fall in GP numbers is "extremely concerning," says BMA. BMJ 2018;361:k2175.

2 Curran A, Parle J. Physician associates in general practice: what is their role? Br J Gen Pract 2018:68:310-1.

3 McMahon T, Barrett T, O'Neill G. Using observation of teaching to improve quality: finding your way through the muddle of competing conceptions, confusion of practice and mutually exclusive intentions. Teaching Higher Educ 2007;12:499-511.

4 Taylor C, Parsons ], Sparrow N, Gerada C. The First5 Concept. Br J Gen Pract 2011;61:72-3.

5 Faculty of Physician Associates. The Faculty of Physician Associates at the Royal College of Physicians. www.fparcp.co.uk [Accessed 16 April 2020].

6 Health Education Yorkshire and Humber. Trainees experiencing difficulties. Health Education England. www.yorksandhumberdeanery. nhs.uk/general_practice/educators/trainees-experiencing-difficulties [Accessed 16 April 2020].

7 Crossley JGM. Addressing learner disorientation: give them a roadmap. Med Teach 2014;36:685-91.

8 Williams LE, Ritsema TS. Satisfaction of doctors with the role of physician associates. Clin Med 2014;14:113-6.

9 Howie N. Continuing professional development for physician associates in primary care. Educ Prim Care 2017;28:197-200. 
10 Worrall P, Scarborough N, Jennings T, Kuhn I. GP-VTS half day release: evaluating a virtual group approach. Educ Prim Care 2006:17:235-43.

11 Royal College of General Practitioners. GP curriculum: overview. London: RCGP. www.rcgp.org.uk/training-exams/training/gpcurriculum-overview.aspx [Accessed 16 April 2020].
Address for correspondence: Mrs Julie Hoskin, NHS Sheffield Clinical Commissioning Group, 722 Prince of Wales Road, Darnall, Sheffield S9 4EU, UK.

Email: juliehoskin@nhs.net

Twitter: @riaagarwal19 